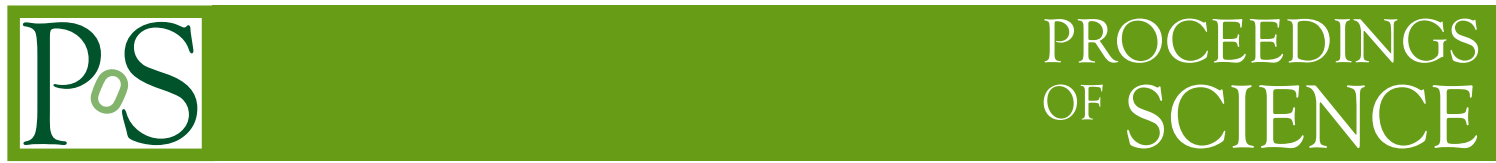

\title{
Lattice QCD with optimal domain-wall fermion on the $20^{3} \times 40$ lattice
}

\author{
Ting-Wai Chiu*1,2,3, Tung-Han Hsieh ${ }^{4}$ (for the TWQCD Collaboration) \\ ${ }^{1}$ Physics Department, National Taiwan University, Taipei 10617, Taiwan \\ ${ }^{2}$ Center for Quantum Science and Engineering, National Taiwan University, \\ Taipei 10617, Taiwan \\ ${ }^{3}$ Center for Theoretical Sciences, National Taiwan University, Taipei 10617, Taiwan \\ ${ }^{4}$ Research Center for Applied Sciences, Academia Sinica, Taipei 115, Taiwan
}

\begin{abstract}
We perform hybrid Monte Carlo simulations of 2 flavors lattice QCD with optimal domain-wall fermion on the $20^{3} \times 40$ lattice, with plaquette gauge action at $\beta=5.95$, for 6 sea-quark masses corresponding to pion masses in the range $230-450 \mathrm{MeV}$. For each sea quark mass, 5000 trajectories are generated after thermalization, and one configuration is sampled every 10 trajectories. In this talk, we present our preliminary results of the topological charge, and the pseudoscalar mass and decay constant.
\end{abstract}

The 30th International Symposium on Lattice Field Theory

June 24-29, 2012

Cairns, Australia

\footnotetext{
*Speaker.
} 


\section{Introduction}

Lattice QCD with exact chiral symmetry [ [, ] ] is an ideal theoretical framework to study the nonperturbative physics from the first principles of QCD. However, it is rather nontrivial to perform Monte Carlo simulation such that the chiral symmetry is preserved at a high precision and all topological sectors are sampled ergodically.

Currently, there are only three groups (RBC/UKQCD, JLQCD, TWQCD) performing largescale dynamical simulations of lattice QCD with exact chiral symmetry. Since the computational requirement for these dynamical simulations is $10-100$ times of their counterparts using the traditional lattice fermions (e.g., Wilson fermion, staggered fermion, and their variants), they are often performed with the state-of-the-art architectures. While RBC/UKQCD and JLQCD have been using IBM Blue Gene supercomputers, TWQCD collaboration has been using a GPU cluster (currently consisting of 320 Nvidia GPUs, with sustained 100 Tflop/s).

The RBC/UKQCD Collaborations have been using the conventional domain-wall fermion with the Shamir kernel [3], 团], which suffers from large chiral symmetry breaking (i.e., large residual mass), especially in the finite temperature QCD. On the other hand, the JLQCD collaboration used the overlap fermion in a fixed topology [ $[$ ], which attains very good chiral symmetry but in the expense of sampling all topological sectors ergodically. To overcome the deficiencies of above two approaches, TWQCD collaboration has been using the optimal domain-wall fermion (ODWF) [ [6, 团] to preserve the chiral symmetry, which not only attains a good chiral symmetry with a modest extension (e.g., $N_{s}=16$ ) in the fifth dimension, but also samples all topological sectors ergodically.

Mathematically, ODWF is a theoretical framework to preserve the chiral symmetry optimally with a set of analytical weights, $\left\{\omega_{s}, s=1, \cdots, N_{s}\right\}$, one for each layer in the fifth dimension [焑]. Thus the artifacts due to the chiral symmetry breaking with finite $N_{s}$ can be reduced to the minimum, especially in the chiral regime. In general, the 4-dimensional effective Dirac operator of massless ODWF can be written as []]

$$
\begin{aligned}
& D=\left[1+\gamma_{5} S_{\text {opt }}(H)\right] /(2 r), \quad S_{\text {opt }}(H)=\frac{1-\prod_{s=1}^{N_{s}} T_{s}}{1+\prod_{s=1}^{N_{s}} T_{s}}, \\
& T_{s}=\frac{1-\omega_{s} H}{1+\omega_{s} H}, \quad H=c H_{w}\left(1+d \gamma_{5} H_{w}\right)^{-1}, \quad r=\left[2 m_{0}\left(1-d m_{0}\right)\right]^{-1},
\end{aligned}
$$

where $c$ and $d$ are constants, and $H_{w}=\gamma_{5} D_{w}\left(-m_{0}\right)$, with $D_{w}\left(-m_{0}\right)$ the usual Wilson-Dirac operator plus a negative parameter $-m_{0}\left(0<m_{0}<2\right)$. Here $S_{\text {opt }}(H)=H R_{Z}(H)$, where $R_{Z}(H)$ is the Zolotarev optimal rational approximation of $\left(H^{2}\right)^{-1 / 2}$ [Q].

Recently we have demonstrated that it is feasible to perform a large-scale dynamical QCD simulation with ODWF, which not only preserves the chiral symmetry to a good precision, but also samples all topological sectors ergodically [ए]]. To recap, we perform HMC simulations of 2 flavors QCD on a $16^{3} \times 32$ lattice, with ODWF at $N_{s}=16$ and plaquette gauge action at $\beta=5.95$. Then we compute the low-lying eigenmodes of the overlap Dirac operator, and use its index to obtain the topological charge of each gauge configuration, and from which we compute the topological susceptibility for 8 sea-quark masses. Our result of the topological susceptibility agrees with the sea-quark mass dependence predicted by the NLO ChPT [Ш]], and provides the first determination of both the pion decay constant $\left(F_{\pi}=92(12)(2) \mathrm{MeV}\right)$ and the chiral condensate 
$\left(\Sigma^{\overline{\mathrm{MS}}}(2 \mathrm{GeV})=[259(6)(7) \mathrm{MeV}]^{3}\right)$ simultaneously from the topological susceptibility. Furthermore, our recent results of the mass and the decay constant of the pseudoscalar meson [ए2] are also in good agreement with the sea-quark mass dependence predicted by NLO ChPT [1[3], and from which we obtain the low-energy constants $F, \Sigma, \bar{l}_{3}$ and $\bar{l}_{4}$. With the low-energy constants, we determine the average up and down quark mass $\left(m_{u d}^{\overline{\mathrm{MS}}}(2 \mathrm{GeV})=4.17(13)(19) \mathrm{MeV}\right)$, and the chiral condensate $\left(\Sigma^{\overline{\mathrm{MS}}}(2 \mathrm{GeV})=[230(4)(6) \mathrm{MeV}]^{3}\right)$. Our results of the topological susceptibility and the mass and decay constant of the pseudoscalar meson assert that the nonperturbative chiral dynamics of the sea-quarks are under control in our HMC simulations.

Recently we have extended our simulations to a larger lattice $\left(20^{3} \times 40\right)$, with plaquette gauge action at $\beta=5.95$, for 6 sea-quark masses corresponding to pion masses in the range 230-450 $\mathrm{MeV}$. In this talk, we present our preliminary results on the topological charge, as well as the mass and decay constant of the pseudoscalar meson.

\section{Lattice Setup}

Simulations are carried out for two flavors QCD on a $20^{3} \times 40$ lattice, for six sea-quark masses $m_{q} a=0.01,0.02, \cdots 0.06$ respectively. For the quark part, we use ODWF with $c=1, d=0$ (i.e., $\left.H=H_{w}\right), N_{s}=16$, and $\lambda_{\min } / \lambda_{\max }=0.02 / 6.2$. For the gluon part, we use the plaquette action at $\beta=5.95$. An outline of our simulation algorithm and features has been presented in Ref. [ए2].

For each sea-quark mass, we generate the initial 400 trajectories on a single GPU. After discarding 300 trajectories for thermalization, we sample one configuration every 5 trajectories, resulting 20 "seed" configurations for each sea-quark mass. Then we use these seed configurations as the initial configurations for 20 independent simulations on 20 GPUs. Each GPU generates 250 trajectories independently. Thus we accumulate total 5000 trajectories for each sea-quark mass. From the saturation of the binning error of the plaquette, as well as the evolution of the topological charge, we estimate the autocorrelation time to be around 10 trajectories. Thus we sample one configuration every 10 trajectories, then we have 500 configurations for each sea-quark mass. With 120 GPUs, we simulate 6 sea-quark masses concurrently. It takes about 10 months to complete the simulations.

For each configuration, we calculate the zero modes plus 180 conjugate pairs of the lowestlying eigenmodes of the overlap Dirac operator. We outline our procedures as follows. First, we project 250 low-lying eigenmodes of $H_{w}^{2}$, using adaptive thick restart Lanczos algorithm (aTRLan), where each eigenmode has a residual less than $10^{-12}$. Then we approximate the sign function of the overlap operator by the Zolotarev optimal rational approximation with 64 poles, where the coefficients are fixed with $\lambda_{\max }^{2}=(6.4)^{2}$, and $\lambda_{\min }^{2}$ equal to the maximum of the 250 projected eigenvalues of $H_{w}^{2}$. Then the sign function error is less than $10^{-14}$. Using the 250 lowmodes of $H_{w}^{2}$ and the Zolotarev approximation with 64 poles, we use the a-TRLan algorithm again to project the zero modes plus 180 conjugate pairs of the lowest-lying eigenmodes of the overlap operator, where each eigenmode has a residual less than $10^{-12}$. We store all projected eigenmodes for the later use.

We compute the valence quark propagator of the 4D effective Dirac operator with the point source at the origin, and with parameters exactly the same as those of the sea-quarks. 
To measure the chiral symmetry breaking due to finite $N_{s}$, we compute the residual mass according to the formula [8]

$$
m_{r e s}=\left\langle\frac{\operatorname{tr}\left(D_{c}+m_{q}\right)_{0,0}^{-1}}{\operatorname{tr}\left[\left(D_{c}^{\dagger}+m_{q}\right)\left(D_{c}+m_{q}\right)\right]_{0,0}^{-1}}\right\rangle_{\{U\}}-m_{q},
$$

where $\left(D_{c}+m_{q}\right)^{-1}$ denotes the valence quark propagator with $m_{q}$ equal to the sea-quark mass, $\operatorname{tr}$ denotes the trace running over the color and Dirac indices, and the subscript $\{U\}$ denotes averaging over an ensemble of gauge configurations.

In Fig. 四, we plot the residual mass versus the sea quark mass. Using the linear fit, we obtain the residual mass in the chiral limit, $m_{\text {res }} a=0.00050(3)$, about $5 \%$ of the lightest sea-quark mass. In the following, it is understood that each bare sea-quark mass $m_{q}$ is corrected by its residual mass, i.e., $m_{q} \rightarrow m_{q}+m_{\text {res }}$.

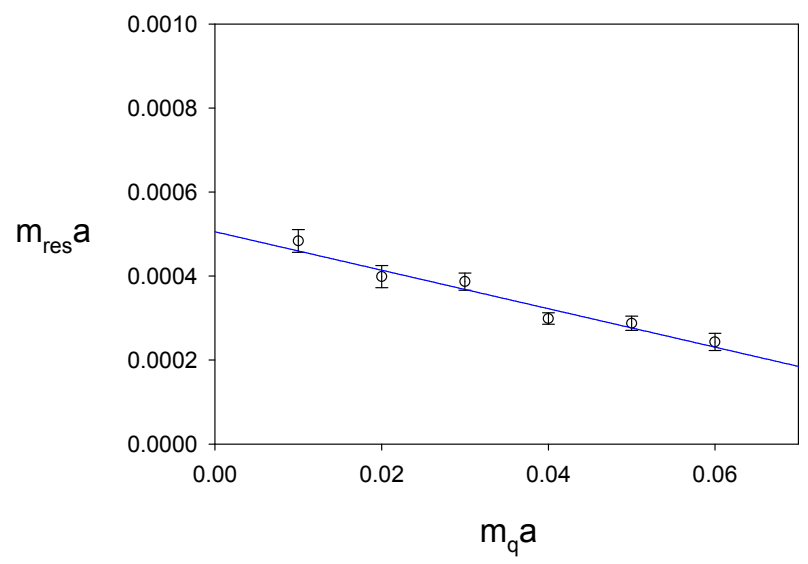

Figure 1: The residual mass versus the sea quark mass for 2-flavor QCD with ODWF.

\section{Preliminary results}

For the projection of eigenmodes (zero modes plus 180 pairs of lowest-lying eigenmodes) of the overlap operator for each configuration, we have only completed about half of the total $(500 \times 6=3000)$ configurations. In Fig. 凤, we plot the histogram of topological charge distribution for $m_{q} a=0.01,0.02, \cdots, 0.06$ respectively. Evidently, the probability distribution of $Q_{t}$ for each sea-quark mass behaves like a Gaussian, and it becomes more sharply peaked around $Q_{t}=0$ as the sea-quark mass $m_{q}$ gets smaller. We will measure the topological susceptibility and other related physical quantities after the projections of all 3000 configurations are completed.

Using the valence quark propgator with quark mass equal to the sea-quark mass, we compute the time-correlation function of the pseudoscalar interpolator

$$
C_{\pi}(t)=\sum_{\vec{x}} \operatorname{tr}\left\{\gamma_{5}\left(D_{c}+m_{q}\right)_{0, x}^{-1} \gamma_{5}\left(D_{c}+m_{q}\right)_{x, 0}^{-1}\right\}
$$



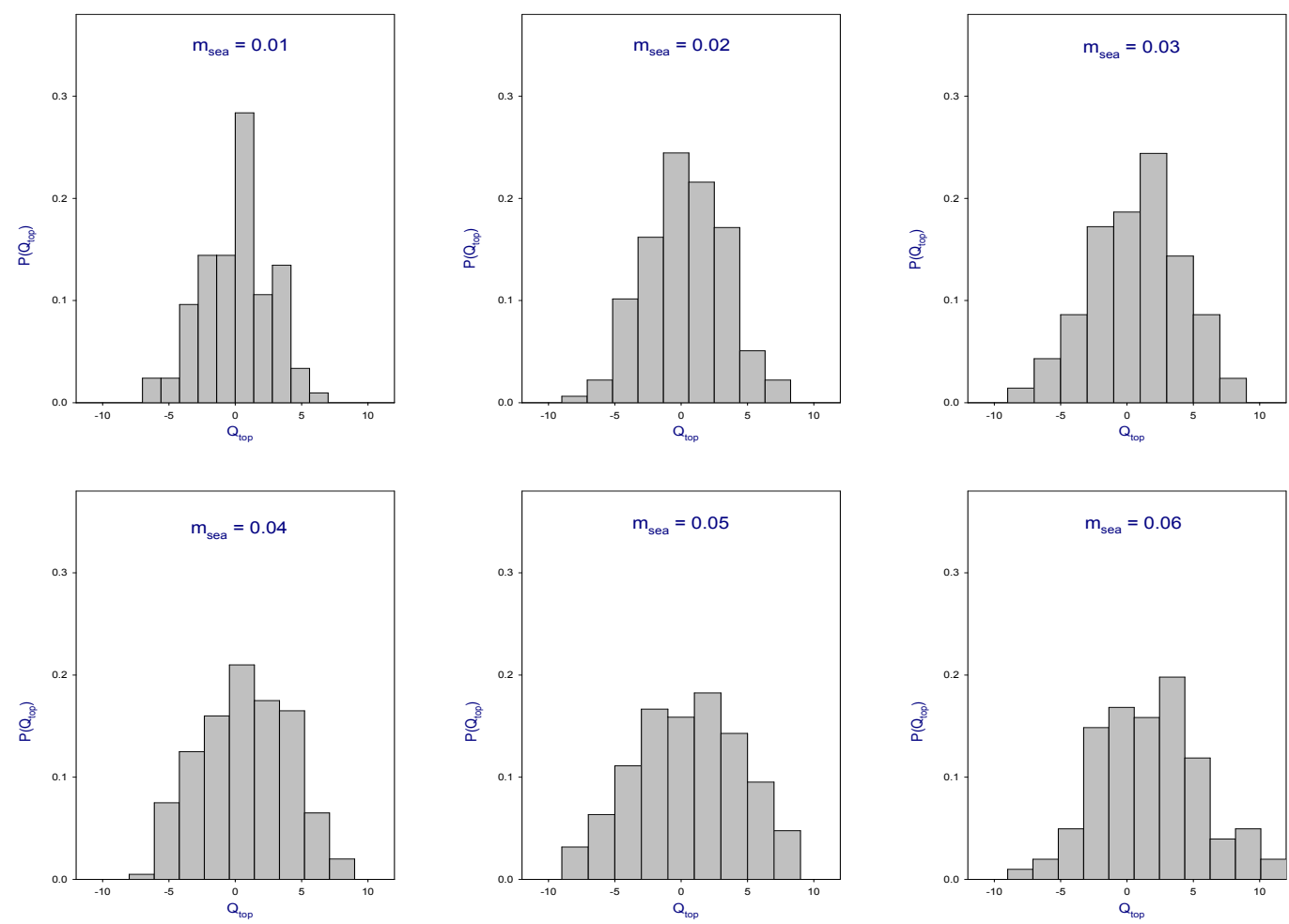

Figure 2: Histogram of topological charge distribution for six sea-quark masses (preliminary results with $\sim 200$ configurations for each ensemble).

where the trace runs over the Dirac and color space. Then the ensemble average $\left\langle C_{\pi}(t)\right\rangle$ of each $m_{q}$ is fitted to the formula $\left(Z /\left(2 M_{\pi} a\right)\right)\left[e^{-M_{\pi} a t}+e^{-M_{\pi} a(T-t)}\right]$ to extract the pion mass $M_{\pi} a$ and the decay constant $F_{\pi} a=\left(m_{q} a \sqrt{2 Z}\right) /\left(M_{\pi}^{2} a^{2}\right)$.

In Fig. 目, we plot $\left(M_{\pi} a\right)^{2} /\left(m_{q} a\right)$ and $F_{\pi} a$ versus $m_{q} a$ respectively. Here we have made the correction for the finite volume effect using the estimate within ChPT calculated up to $\mathscr{O}\left(M_{\pi}^{4} /\left(4 \pi F_{\pi}\right)^{4}\right)$ [प14]. Taking into account of the correlation between $M_{\pi}^{2} / m_{q}$ and $F_{\pi}$ for the same sea-quark mass, we fit our data to the formulas of NLO ChPT [13]]

$$
\begin{aligned}
\frac{M_{\pi}^{2}}{m_{q}} & =\frac{2 \Sigma}{F^{2}}\left[1+\left(\frac{\Sigma m_{q}}{16 \pi^{2} F^{4}}\right) \ln \left(\frac{2 \Sigma m_{q}}{F^{2} \Lambda_{3}^{2}}\right)\right], \\
F_{\pi} & =F\left[1-\left(\frac{\Sigma m_{q}}{8 \pi^{2} F^{4}}\right) \ln \left(\frac{2 \Sigma m_{q}}{F^{2} \Lambda_{4}^{2}}\right)\right],
\end{aligned}
$$

where $\Lambda_{3}$ and $\Lambda_{4}$ are related to the low energy constants $\bar{l}_{3}$ and $\bar{l}_{4}$ as follows.

$$
\bar{l}_{3}=\ln \left(\frac{\Lambda_{3}^{2}}{m_{\pi^{ \pm}}^{2}}\right), \quad \bar{l}_{4}=\ln \left(\frac{\Lambda_{4}^{2}}{m_{\pi^{ \pm}}^{2}}\right), \quad m_{\pi^{ \pm}}=0.140 \mathrm{GeV}
$$

Our procedure of data fitting to extract the parameters $\left(\Sigma, F, \Lambda_{3}\right.$ and $\left.\Lambda_{4}\right)$ has been outlined in 


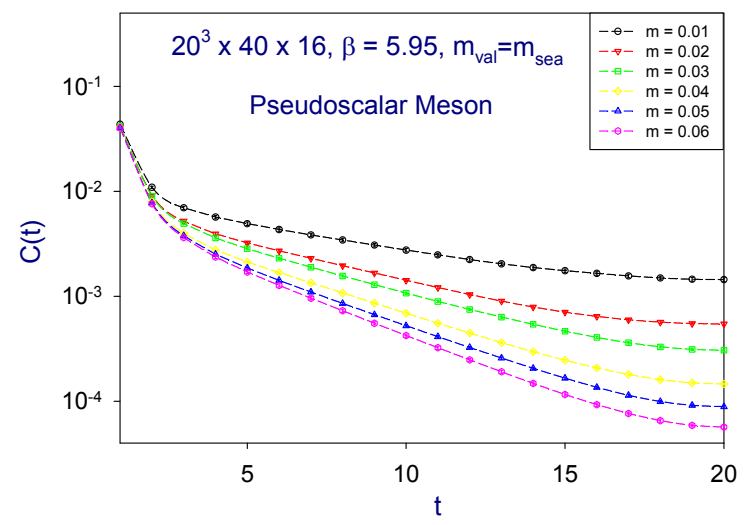

(a)

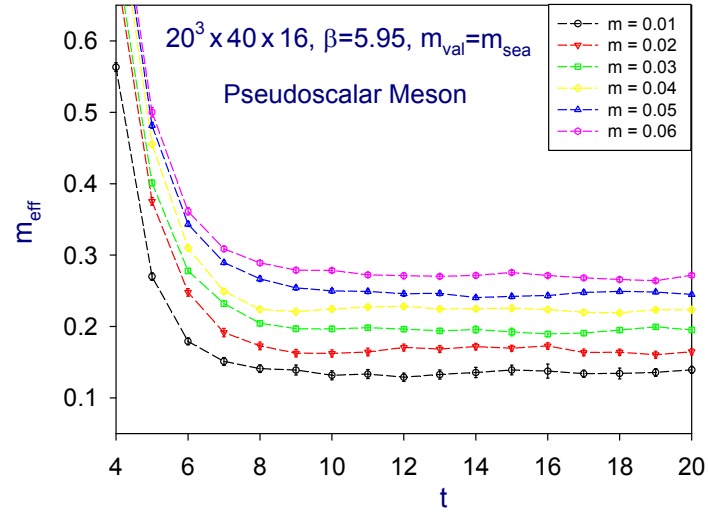

(b)

Figure 3: (color online) (a) The time-correlation function of the pseudoscalar meson for six sea quark masses. (b) The effective mass of (a). The dashed line connecting the data points of the same sea-quark mass is for guiding the eyes.

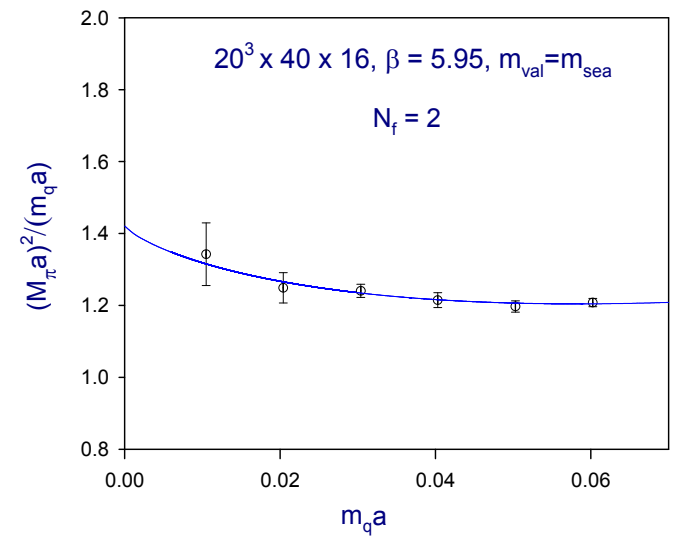

(a)

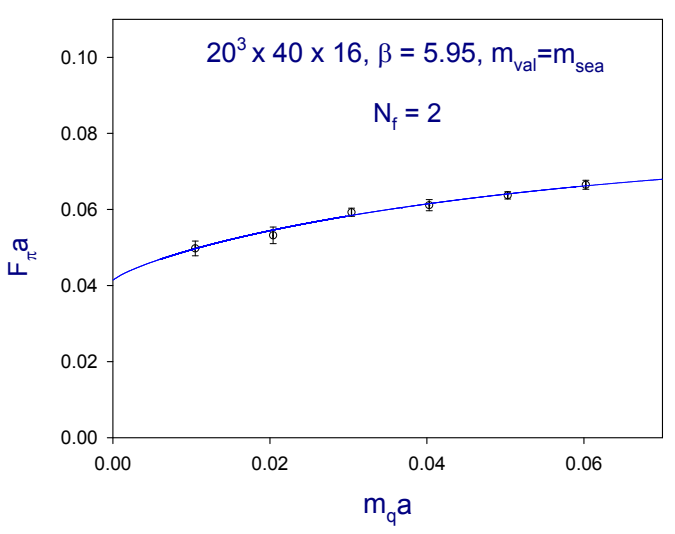

(b)

Figure 4: Pseudoscalar meson of 2 flavors QCD with ODWF (a) $\left(M_{\pi} a\right)^{2} /\left(m_{q} a\right)$, and (b) $F_{\pi} a$. The solid lines are the simultaneous fits to the NLO ChPT, for six sea-quark masses.

Ref. [[2]]. For six sea-quark masses, our fit gives

$$
\begin{array}{ll}
\Sigma a^{3}=0.00122(6)(2), & F a=0.0414(10)(16), \\
\bar{l}_{3}=3.829(105)(43), & \bar{l}_{4}=4.755(93)(23),
\end{array}
$$

where the systematic errors are estimated by varying the number of data points from 6 to 4 ( $m_{q} a \leq$ 0.04 ).

With the fitted parameters, we use the physical ratio

$$
\left(\frac{M_{\pi}}{F_{\pi}}\right)^{\text {phys }}=\frac{0.135 \mathrm{GeV}}{0.093 \mathrm{GeV}} \simeq 1.45
$$


as the input, and solve the equation $M_{\pi}\left(m_{q}\right) / F_{\pi}\left(m_{q}\right)=1.45$ to obtain the physical bare quark mass $m_{q}^{\text {phys }} a=0.00254(10)(16)$. From (B.2) and the physical pion decay constant $F_{\pi}=92.6 \mathrm{MeV}$, we determine the inverse lattice spacing at the physical point,

$$
1 / a=2.076(6)(5) \mathrm{GeV} \text {. }
$$

From (B.]), we obtain the pion mass at the physical point, $M_{\pi}=0.134(5)(3) \mathrm{GeV}$, which serves as a consistency check.

In order to convert the chiral condensate $\Sigma$ and the average $m_{u}$ and $m_{d}$ to those in the $\overline{\mathrm{MS}}$ scheme, we calculate the renormalization factor $Z_{s}^{\overline{\mathrm{MS}}}(2 \mathrm{GeV})$ using the non-perturbative renormalization technique through the RI/MOM scheme [प15], which gives $Z_{s}^{\overline{\mathrm{MS}}}(2 \mathrm{GeV})=1.244(18)(39)$. Then the values of $\Sigma$ and the average of $m_{u}$ and $m_{d}$ are transcribed to

$$
\begin{aligned}
& \Sigma^{\overline{\mathrm{MS}}}(2 \mathrm{GeV})=[238(10)(6) \mathrm{MeV}]^{3}, \\
& m_{u d}^{\overline{\mathrm{MS}}}(2 \mathrm{GeV})=4.07(13)(12) \mathrm{MeV} .
\end{aligned}
$$

Our preliminary results of the chiral condensate (B.4) and the average up and down quark mass (B.5) are in good agreement with our previous results on the $16^{3} \times 32$ lattice [12].

This work is supported in part by the National Science Council (Nos. NSC99-2112-M-002012-MY3, NSC99-2112-M-001-014-MY3) and NTU-CQSE (No. 10R80914-4). We also thank NCHC and NTU-CC for providing facilities to perform part of our calculations.

\section{References}

[1] D. B. Kaplan, Phys. Lett. B 288, 342 (1992); Nucl. Phys. Proc. Suppl. 30, 597 (1993).

[2] H. Neuberger, Phys. Lett. B 417, 141 (1998); R. Narayanan and H. Neuberger, Nucl. Phys. B 443, 305 (1995).

[3] Y. Shamir, Nucl. Phys. B 406, 90 (1993)

[4] C. Allton et al. [RBC and UKQCD Collaboration], Phys. Rev. D 76, 014504 (2007)

[5] H. Fukaya et al. [JLQCD Collaboration], Phys. Rev. D 74, 094505 (2006)

[6] T. W. Chiu, Phys. Rev. Lett. 90, 071601 (2003); Phys. Lett. B 552, 97 (2003); Phys. Lett. B 716, 461 (2012); Nucl. Phys. Proc. Suppl. 129, 135 (2004)

[7] T. W. Chiu et al. [TWQCD Collaboration], PoS LAT2009, 034 (2009).

[8] Y. -C. Chen and T. W. Chiu [TWQCD Collaboration], Phys. Rev. D 86, 094508 (2012)

[9] T. W. Chiu, T. H. Hsieh, C. H. Huang and T. R. Huang, Phys. Rev. D 66, 114502 (2002).

[10] T. W. Chiu, T. H. Hsieh and Y. Y. Mao [TWQCD Collaboration], Phys. Lett. B 702, 131 (2011).

[11] Y. Y. Mao and T. W. Chiu [TWQCD Collaboration], Phys. Rev. D 80, 034502 (2009)

[12] T. W. Chiu, T. H. Hsieh and Y. Y. Mao [TWQCD Collaboration], Phys. Lett. B 717, 420 (2012)

[13] J. Gasser and H. Leutwyler, Nucl. Phys. B 250, 465 (1985).

[14] G. Colangelo, S. Durr and C. Haefeli, Nucl. Phys. B 721, 136 (2005).

[15] G. Martinelli, C. Pittori, C. T. Sachrajda, M. Testa and A. Vladikas, Nucl. Phys. B 445, 81 (1995). 